\title{
Traduire
}

Revue française de la traduction

234 | 2016

La traduction, un sport de haut niveau

\section{La passion, la tête et les jambes}

Idriss Chaplain

\section{(2) OpenEdition}

\section{Journals}

Édition électronique

URL : http://journals.openedition.org/traduire/804

DOI : 10.4000/traduire.804

ISSN : 2272-9992

Éditeur

Société française des traducteurs

Édition imprimée

Date de publication : 15 juin 2016

Pagination : 60-63

ISSN : 0395-773X

\section{Référence électronique}

Idriss Chaplain, «La passion, la tête et les jambes », Traduire [En ligne], 234 | 2016, mis en ligne le 15 juin 2018, consulté le 20 avril 2019. URL : http://journals.openedition.org/traduire/804 ; DOI :

10.4000/traduire.804 


\section{La passion, la tête et les jambes}

\section{Idriss Chaplain}

Peut-être êtes-vous de ceux qui, comme moi, ne voient pas la traduction comme un domaine, mais un ensemble d'infinis sous-domaines. La traduction sportive, " mon " domaine, consiste simplement à traduire des ouvrages en lien avec le sport. Ma façon de penser la traduction est la même que ma façon d'aborder la vie en général : peu importe nos compétences, le seul moteur qui vaille reste la passion. Pour moi, choisir une spécialisation juste parce qu'elle offre " plus de débouchés" ou qu'il y a " toujours du travail " n'a jamais été une option et ne peut mener nulle part, sur le long terme.

\section{La passion : condition sine qua non}

C'est donc par passion que, tout au long de mon cursus (Master 1 général en 2012-13, et Master 2 juridique, économique et technique en 2013-14), j'ai délibérément choisi d'orienter le moindre projet de traduction libre vers le sport. Mon mémoire de Master 1 consistait en un glossaire français-anglais du floorball ; pour celui de Master 2, j'ai choisi de traduire (partiellement) un ouvrage de rugby. Je me souviens aussi d'un projet de retraduction qui m'avait permis de revoir et corriger la version anglaise d'un appareil de musculation. Ces choix se sont avérés payants puisqu'à l'heure actuelle, j'ai la chance de vivre exclusivement de la traduction sportive (pourvu que cela dure).

C'est lors de mon stage de fin de Master 2 que j'ai eu la chance de rencontrer celui qui m'a mis le pied à l'étrier dans ce milieu : Laurent Laget(1). II animait alors un colloque organisé par la SFT sur le thème de la traduction sportive - et ce fut une révélation.

Ce jour-là, Laurent a présenté les tenants et les aboutissants de sa profession. II a fait une réflexion qui m'a profondément marqué, d'ailleurs plus par la réaction qu'elle a suscitée chez

(1) Voir l'article "Le cauchemar des interprètes ", page 18 de ce numéro. 
les traducteurs qui m'entouraient, pourtant tous aguerris, que par son contenu. En effet, quand il a dit qu'il était courant pour un traducteur sportif de traduire en moyenne 10000 mots par jour, tout le monde a eu un regard ébahi. Je comprenais leur réaction, car la plupart de mes professeurs d'alors situaient le nombre de mots à traduire au quotidien, pour un traducteur professionnel, entre 2000 et 4000 maximum. Si je n'étais moi-même pas encore professionnel, cela ne m'a pas étonné, car je savais déjà que ce qui ralentit un traducteur, c'est la quantité de recherches qu'il doit effectuer quand il traite un document.

Pour le traducteur sportif, c'est différent ; en effet, il ne passe que très peu de temps à faire des recherches annexes. Soit il travaille sur un texte traitant d'un sport dont il est spécialiste, auquel cas il maîtrise déjà tout le vocabulaire. Soit il traduit un document relatif à une discipline sportive dont il ignore les complexités, mais en assimilera rapidement les formulations. Dans les deux cas, il lui faudra passer beaucoup moins de temps autour du texte que n'importe quel traducteur abordant un domaine qui lui est inconnu. J'ai donc pu constater que les propos de Laurent étaient exacts, tant concernant l'importance du volume traité, que le fait qu'il n'était pas forcément plus indigeste qu'un faible volume dont nous ne maîtrisons pas la teneur.

\section{Au fil du temps...}

À cette évocation, je repense toujours à l'un des (peu nombreux) textes à teneur sportive que nous avons dû traduire lors de mon année de Master 2. II s'agissait d'un article de journal résumant un match de football : une des phrases anglaises comprenait l'expression clean sheet (autrement dit le fait qu'une équipe termine le match sans encaisser de but). Mes camarades, pour qui l'univers du football était complètement opaque, ont, bien entendu, cherché la signification de ce terme sur internet et dans les dictionnaires. Ils ont trouvé pour traduction le mot français "blanchissage ", qu'ils n'ont pas hésité à inclure dans leur copie. Seulement, tout amateur de sport, et plus particulièrement de football, sait que ce terme, bien qu'avéré, n'est jamais utilisé dans ce contexte. En effet, en français, on ne parlera de "blanchissage " que dans les sports de crosse, de type hockey sur glace. Quand il s'agit de football, on dira simplement que "le gardien a gardé sa cage inviolée ", ou une tournure équivalente.

Cet exemple incarne à merveille cette " fibre " impalpable qui anime un traducteur sportif. Plus que tout autre sous-domaine, la traduction sportive répond à des codes que seuls les sportifs peuvent comprendre. Au fil des années, et des traductions, on peut devenir traducteur juridique, traducteur touristique, etc. Pour le sport, c'est différent. II s'agit d'une véritable passion. Souvent, on baigne dedans depuis l'enfance et, comme on assimile sa langue maternelle, on acquiert naturellement les codes du jargon et de la culture du sport, par amour. 


\section{Déformation(s) professionnelle(s)}

Face à cette double passion (sport et traduction) et à cette activité professionnelle si peu commune, on me demande parfois si le fait d'être sportif (entre 10 et 20 heures de pratique hebdomadaire) influence ma façon de traduire et si, à l'inverse, les textes que je traduis ont un impact sur ma façon de m'entraîner.

La question est pertinente. Comme j'ai pu l'expliquer plus haut, le fait d'être sportif présente deux avantages : le premier est celui de gagner un temps considérable quand on traite des volumes en rapport à des disciplines que l'on maîtrise. Le second, et je l'ai constaté au fil du temps, est que, face à des disciplines sportives qui nous sont inconnues, on assimile le jargon bien plus facilement, et les tournures de phrases nous viennent naturellement.

Mais cela fonctionne aussi dans l'autre sens ; en effet, mon métier a une influence sur ma pratique sportive. Des nombreux textes qu'il m'arrive de traiter (en particulier les programmes d'entraînement et les conseils de nutrition), je tire souvent des enseignements très intéressants que je choisis parfois de mettre en pratique. De plus, cela me permet de développer une curiosité et une passion nouvelles. Ainsi, le fait de traduire l'autobiographie de Chris Froome, le vainqueur du Tour de France, m'a donné l'envie, pour la première fois de ma vie, de suivre la Grande Boucle cet été. J'ai également pris l'habitude de faire plus de vélo de course, quand je vais m'entraîner à la salle de sport (le syndrome de Stockholm, sans doute...).

J'ai volontairement insisté sur mes années d'étudiant dans ce témoignage car il me semble que le sport est un domaine encore trop peu considéré, voire dénigré, au sein des cursus de traduction. J'en veux pour preuve le peu de textes sportifs que j'ai eu à traduire au cours de mon cursus, et l'attitude hautaine de certains de mes professeurs à l'évocation du sport, lorsque nous corrigions nos textes, à Lille. Nous travaillions régulièrement sur des textes relatifs à la médecine, à la cuisine, au tourisme, ou encore à la gastronomie; nous avions même la chance de pouvoir suivre un cours de traduction de jeux vidéo. Le sport, lui, était un domaine presque totalement éludé. Même sans faire forcément l'objet d'un cours à part entière (comme les jeux vidéo), le sport a un poids considérable dans la société d'aujourd'hui, et les études littéraires ont parfois tendance à l'oublier. II est vecteur d'émotions, et permet de se rassembler, de partager, de se retrouver, et même d'oublier les tracas du quotidien.

Aujourd'hui, quand je traduis des articles, comptes-rendus ou livres relatifs au sport, j'ai le sentiment de me faire le relais de cette passion. Et c'est plus qu'un privilège. C'est une fierté. 
Idriss Chaplain a commencé son cursus en LLCE à l'université de Caen en 2009, puis validé sa licence en Erasmus à l'University College of Cork, en Irlande. II a ensuite effectué une première année de Master MéLexTra à l'université de Lille III, puis validé son Master 2 spécialité Juridique, Économique et Technique. Installé comme freelance depuis 2014, il travaille principalement pour I'UEFA (via la version française du site uefa.com, le compte Facebook UEFA Champions League, ou encore le compte Twitter@UEFAcom_fr), le magazine mensuel Men's Fitness (anciennement Men's Health), les Éditions Vigot (pour qui il a traduit TRIATHLON : 100 séances d'entraînement et La science du tennis - à paraitre) et les Éditions Solar (pour qui il a traduit l'autobiographie de Chris Froome, vainqueur du Tour de France, à paraitre courant 2016).

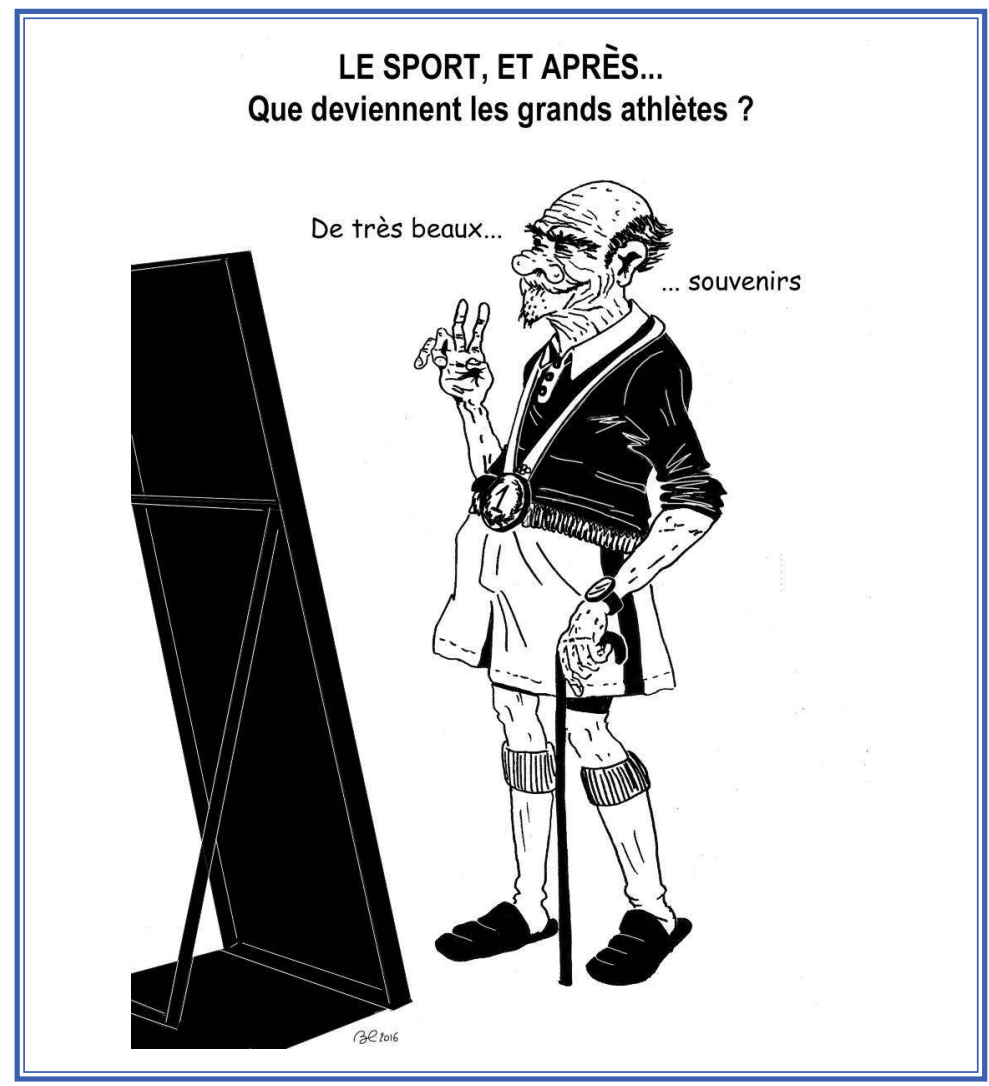

